\title{
Fuzzy logic feedback control for fed-batch enzymatic hydrolysis of lignocellulosic biomass
}

\author{
Chao Tai ${ }^{1} \cdot$ Diego S. Voltan ${ }^{1,2} \cdot$ Deepak R. Keshwani ${ }^{1} \cdot$ George E. Meyer $^{1} \cdot$ \\ Pankaj S. Kuhar ${ }^{1}$
}

Received: 17 November 2015 / Accepted: 15 February 2016/Published online: 25 February 2016

(C) Springer-Verlag Berlin Heidelberg 2016

\begin{abstract}
A fuzzy logic feedback control system was developed for process monitoring and feeding control in fed-batch enzymatic hydrolysis of a lignocellulosic biomass, dilute acid-pretreated corn stover. Digested glucose from hydrolysis reaction was assigned as input while doser feeding time and speed of pretreated biomass were responses from fuzzy logic control system. Membership functions for these three variables and rule-base were created based on batch hydrolysis data. The system response was first tested in LabVIEW environment then the performance was evaluated through real-time hydrolysis reaction. The feeding operations were determined timely by fuzzy logic control system and efficient responses were shown to plateau phases during hydrolysis. Feeding of proper amount of cellulose and maintaining solids content was well balanced. Fuzzy logic proved to be a robust and effective online feeding control tool for fed-batch enzymatic hydrolysis.
\end{abstract}

Deepak R. Keshwani

dkeshwani2@unl.edu

Chao Tai

mtai@icloud.com

Diego S. Voltan

diegosvoltan@gmail.com

George E. Meyer

gmeyer1@unl.edu

Pankaj S. Kuhar

pankajsinghkuhar@yahoo.in

1 Department of Biological Systems Engineering, University of Nebraska-Lincoln, Lincoln, NE 68583, USA

2 Rural Engineering Department, College of Agricultural Sciences, São Paulo State University, Botucatu, SP 18610-307, Brazil
Keywords Fuzzy logic - Enzymatic hydrolysis · Feedback control · Bioconversion · Biofuels

\section{Introduction}

The bioconversion of cellulosic biomass to ethanol is one technology currently being assessed for its potential to supplement the use of current fossil fuel-derived gas [1, 2]. In this process, cellulosic biomass will be pretreated before enzymatic hydrolysis to allow easier access of enzyme to targeted substrate. The monosaccharides (mostly glucose) derived from hydrolysis will then be used for fermentation to produce ethanol, which will be followed by distillation to get concentrated ethanol product. It has been noted that the enzyme cost during hydrolysis process and energy cost during ethanol distillation process are two of the highest major cost in the whole work [3, 4]. It has been estimated that, by doubling the ethanol concentration from 2.5 to $5 \%$, the energy required to distill a fermentation broth to $93.5 \%$ ethanol with conventional distillation techniques can be reduced by about $33 \%$ [5]. Ethanol concentration in fermentation broth is strongly correlated to the sugar concentration which is available for fermentation [6]. Hence, higher sugar concentrations are desirable. Fed-batch hydrolysis is a potential strategy to achieve this while reducing the challenges associated with high solid loadings during hydrolysis [7]. Fed-batch studies have been shown to improve sugar yields in some previous work [8] while some work has also suggested that the fedbatch approach is no better than the traditional single batch approach $[9,10]$. The reported differences in the various fedbatch hydrolysis results are likely due to the fact that, both the quantities and timing of pretreated substrate and enzyme that are added to the actively hydrolyzing reaction are critical. These two factors have significant direct influence on the 
active enzyme transition between substrates and new adsorption equilibrium [11].

Online monitoring and control technologies have been studied to identify critical time points and proper feeding operations, but ambiguity is always present in any realistic process [12, 13]. Precise indexes such as active enzyme quantity and resided substrate in system are challenging to be obtained online to build feeding responses. The influence of added pretreated substrate and enzyme on the actively hydrolysis reaction is hard to be perfectly predicted by existing models. Hence, adjusting the feeding operation is not a trivial task due to ambiguity.

Fuzzy logic, which may be viewed as an extension of classical logical systems, provides an effective conceptual framework for dealing with the problem in an environment of uncertainty and imprecision [14]. The logic underlying mode of reasoning which is approximate rather than exact provides a promising approach where ambiguity regarding the system parameters is present. Linguistic variables are often used to handle the concept of partial truth such as "warm" in the range between "cold" and "hot". It is one technology that has been validated as a promising control strategy in these practical ambiguity applications such as continuous digestion process, dissolved oxygen control in reactor, and waste water treatment [15-17]. With the advantage of dealing with imprecision, relatively less information may be needed for fuzzy logic control system to generate reasonable control operations as responses.

In this study, a fuzzy logic control system was built to monitor the enzymatic hydrolysis reaction and control feeding operations for a fed-batch approach. Glucose concentration from hydrolysis was initially analyzed online and digested glucose was assigned as input while feeding time and speed of biomass were responses in a fuzzy logic control rule-base system. The performance of fuzzy logic control was evaluated for fed-batch enzymatic hydrolysis.

\section{Materials and methods}

\section{Materials}

Corn stover was collected from Rogers Memorial Farm (Lincoln, NE, USA) in 2012, it was air dried, milled, screened through $2.36 \mathrm{~mm}$ sieve and homogenized in a single lot. The enzyme preparation used in this work was Cellic CTec2 which was kindly provided by Novozymes North America Inc. (Franklinton, NC, USA).

\section{Pretreatment}

Corn stover samples were pretreated with $1.75 \%(\mathrm{w} / \mathrm{v})$ sulfuric acid in sealed flasks in an autoclave at $135{ }^{\circ} \mathrm{C}$ for
$160 \mathrm{~min}$. Solid to liquid ratio was 1:10. The pretreated biomass recovered by filtration through a porcelain Buchner funnel was washed with distilled water until pH was 7 . The wet solids were completely transferred to a preweighed plastic bag, weighed and stored sealed at $4{ }^{\circ} \mathrm{C}$ for the enzymatic hydrolysis later. A small portion of the wet pretreated biomass was weighed and dried for composition analysis.

\section{Enzymatic hydrolysis}

Batch enzymatic hydrolysis experiments were conducted in $50 \mathrm{~mL}$ total volume in $125 \mathrm{~mL}$ screw-top Erlenmeyer flasks with $10 \%(\mathrm{w} / \mathrm{v})$ dry substrate and $20 \mathrm{FPU} / \mathrm{g}$ cellulose of enzyme $[18,19]$. The activity of CTec 2 enzyme was determined by standard procedure developed by National Renewable Energy Laboratory (NREL) [20]. $0.05 \mathrm{~mol} / \mathrm{L}$ sodium citrate buffer was used to maintain $\mathrm{pH} 5.0$ and tetracycline $(0.004 \%, \mathrm{w} / \mathrm{v})$ and cycloheximide $(0.003 \%$, $\mathrm{w} / \mathrm{v}$ ) were added to the hydrolysis mixture to prevent microbial growth. The hydrolysis was carried out at $50{ }^{\circ} \mathrm{C}$ and $150 \mathrm{rpm}$ for $72 \mathrm{~h}$ in a controlled environmental incubator shaker (Model I26, New Brunswick Scientific, NJ, USA). Aliquots of $0.3 \mathrm{~mL}$ were taken at specified time intervals during hydrolysis and centrifuged at 10,000 rpm for $10 \mathrm{~min}$, the supernatant was used for sugar analysis.

Fed-batch enzymatic hydrolysis experiments were conducted in a $1.3 \mathrm{~L}$ bioreactor (Model BioFlo115, New Brunswick Scientific, NJ, USA) using fuzzy logic control. The system setup is shown in Fig. 1. The hydrolysis started with an initial loading volume of $300 \mathrm{~mL}$. Hydrolysis conditions were the same as in batch hydrolysis. Aliquots of $3 \mathrm{~mL}$ were taken every $2 \mathrm{~h}$ using a SEG-FLOW automated online sampling system (Model 4800, Flownamics, WI, USA), samples were then sent to a biochemistry analyzer (Model 2700, YSI, FL, USA) where the glucose concentration was determined. The glucose concentration results were then sent to a computer (Dell, TX, USA) where the fuzzy logic control program was installed in a LabVIEW environment (version 2012, National Instruments, TX, USA). The feeding rate for pretreated substrate based on the digested glucose was determined by the fuzzy logic control system, while enzyme feeding rate was fixed at $18.5 \mathrm{FPU} / \mathrm{h}$, which was targeted to reach around an average of $20 \mathrm{FPU} / \mathrm{g}$ cellulose at the end of hydrolysis. Acid-pretreated biomass was dried at $50{ }^{\circ} \mathrm{C}$ overnight to be used as pretreated feedings, feeding operation was conducted by a doser (Lambda Lab Instruments, Brno, Czech Republic) which can receive feeding commands from LabVIEW. The enzyme preparation was diluted properly so the feeding volume would be equal to the sample volume. It takes about $30 \mathrm{~s}$ to take the sample and about $60 \mathrm{~s}$ for YSI to analyze. The signal communication time 
Fig. 1 Setup of fed-batch enzymatic hydrolysis with fuzzy logic control system

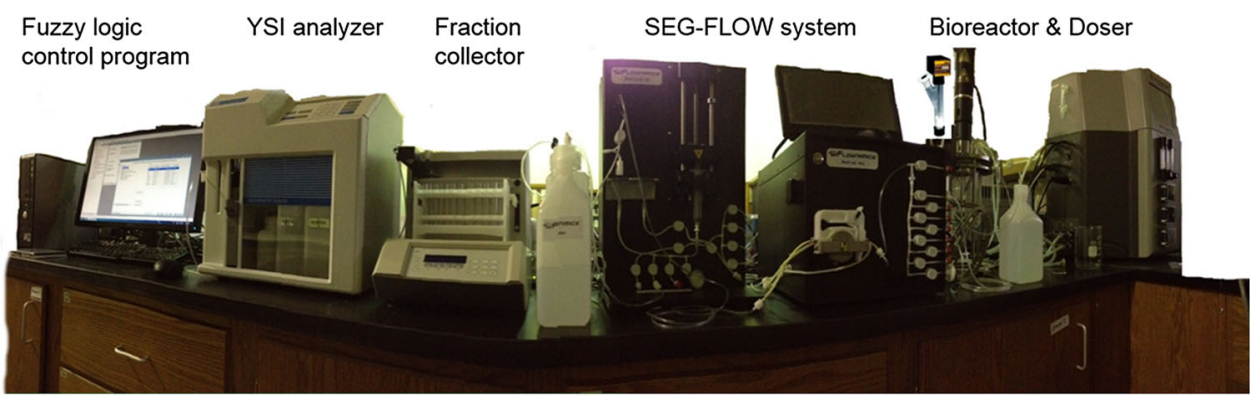

between each equipment and computer is less than $1 \mathrm{~s}$. Since the sampling interval is $2 \mathrm{~h}$, the time needed for the sample operation, analysis and communication is negligible.

\section{Composition and sugar analysis}

Chemical composition of pretreated corn stover was analyzed using standard analytical procedures developed by NREL [21, 22]. Sugars in the batch hydrolysate were measured in a HPLC system (Model Ultimate 3000, Dionex) with a Bio-Rad Aminex HPX-87P column (300 mm $\times 7.8 \mathrm{~mm}$ ), a Bio-Rad de-ashing guard column, and a refractive index detector. The mobile phase was HPLC grade water at a flow rate of $0.6 \mathrm{~mL} / \mathrm{min}$ and the column temperature was $85^{\circ} \mathrm{C}$.

\section{Fuzzy logic control}

The objective of the feedback control in this work was to develop an online control of enzymatic hydrolysis through the action of feeding doser controller based on the signal of glucose concentration in bioreactor. To achieve an efficient control, solids content in system must be maintained and high cellulose conversion rate has to be reached. Proper feeding responses have to be adjusted once the cellulose conversion rate decreases to different levels.

The fuzzy logic control system was built in three steps. The first step was to partition the universe of interval spanned by each variable into several fuzzy subsets with appropriate linguistic labels (e.g., high, medium and low for temperature). Next, a membership function was postulated for each fuzzy subset, followed by assigning the fuzzy relationships between input fuzzy subsets and output fuzzy subsets, thus forming rule-base [23]. In this study, the input variable was digested glucose $(\mathrm{g} / \mathrm{h})$ from hydrolysis while the output variables were doser feeding time length (s) and feeding speed (\%). The feeding speed was set in a range of 0-999. The speed control was tested to dose certain amount of biomass within a certain time frame. The membership functions for the three variables are shown in Fig. 2 and the rule-base between input and output is shown in Table 1, which will be discussed in Sect. 3.1.

The fuzzy logic control system was programmed in LabVIEW environment (version 2012, National Instruments, TX, USA) and part of the program was shown in Fig. 3. When the system was operating, a crisp input of digested glucose was calculated based on glucose concentration signal from biochemistry analyzer. This crisp input is then delineated as to its membership in the input fuzzy sets. With the rules defined between input and output, the fuzzy output was then defuzzified to crisp control values with the center of area method [23]. The numerical

a

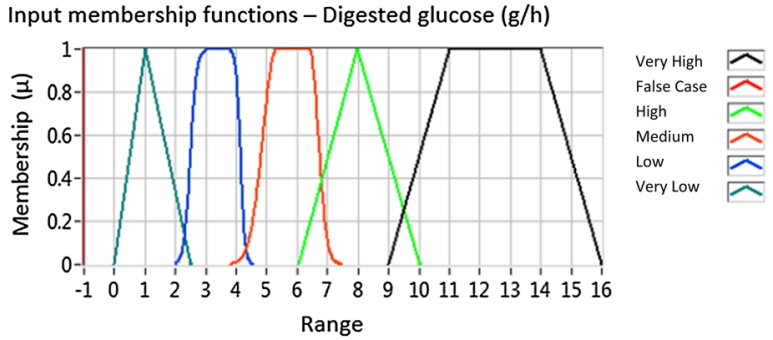

b

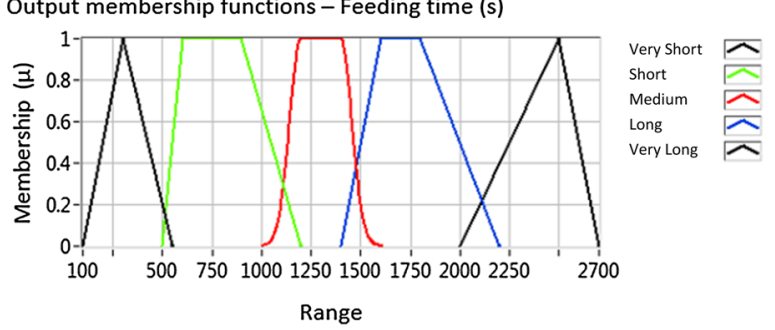

C

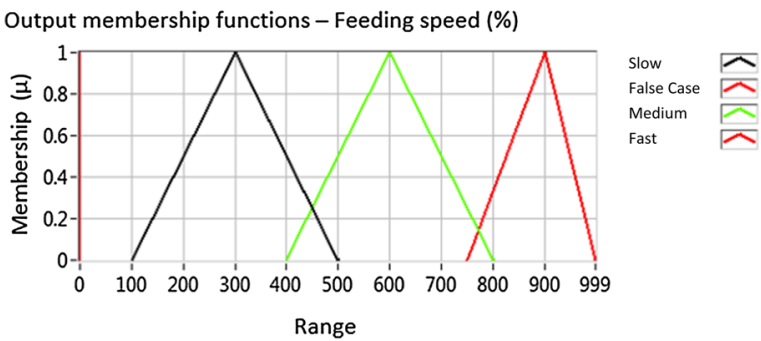

Fig. 2 Membership functions for input and output variables in fuzzy logic control system 
Table 1 Rule-base for input and output variables in fuzzy logic control system

\begin{tabular}{lll}
\hline Input variable & Output variables & \\
\cline { 2 - 3 } Digested glucose $(\mathrm{g} / \mathrm{h})$ & Feeding time $(\mathrm{s})$ & Feeding speed $(\%)$ \\
\hline Very high & Very long & Fast \\
High & Long & Fast \\
High & Very long & Medium \\
Medium & Medium & Fast \\
Medium & Long & Medium \\
Low & Short & Fast \\
Low & Short & Medium \\
Low & Long & Slow \\
Very low & Very short & Fast \\
Very low & Very short & Medium \\
Very low & Short & Slow \\
False case & Very short & False case \\
\hline
\end{tabular}

values of output were eventually sent to doser controller which took action of feeding pretreated substrate.

\section{Results and discussion}

Most of the previous fed-batch hydrolysis studies can be classified in three categories. The first category of studies involves manual feeding operations, where pretreated substrate or enzyme is fed manually at different time intervals during hydrolysis based on offline detection and observation [24, 25]; the second category of studies involves model-based open loop control where the substrate consumption rate is calculated based on kinetic models before operation and a set of pre-determined feeding operations at specified time points would be implemented after hydrolysis starts. No feedback from hydrolysis would be taken and no adjustment would be applied to feeding operation during the actual hydrolysis process [8, 10]; the third category, model-based closed loop control involves feeding operations based on kinetic models, but the process is being monitored online and feedback signals from the hydrolysis are used to correct or adjust feeding operations for optimum control [26].

Manual feeding operations apparently require more labor work while the feeding responses could not be timely enough because of the relatively long time intervals. While model-based closed loop control is more favorable there are still obstacles such as obtaining appropriate indexes in hydrolysis system for model calculation and precise models to correctly reflect the influence of newly added pretreated feedings on active hydrolysis system. With the drawbacks described above, fuzzy logic control provides the advantages of a data-driven process combined with timely adjustment of operations where glucose release rate is sufficient to represent the hydrolysis system ongoing situation, and the feeding operations are determined with the consideration of pretreated feedings interference.

\section{Analysis of membership functions and rules}

Based on the batch enzymatic hydrolysis data, the input membership functions of digested glucose were defined in the range from 0 to $16 \mathrm{~g} / \mathrm{h}$ (Fig. 2a). A sharp triangle type membership was assigned to "Very Low" status with a range from 0 to $2.5 \mathrm{~g} / \mathrm{h}$. Gaussian type membership was assigned to "Low" and "Medium" with certain overlaps with "Very Low" and "High" so the full membership would cover wider ranges of $2-4.5$ and $3.8-7.5 \mathrm{~g} / \mathrm{h}$, respectively. The overlap between "Medium" and "High" is relatively bigger to give more flexibility to feeding output. Not much feeding action would vary if the digested glucose was high enough so the full membership for "Very High" was set to cover the range of $11-16 \mathrm{~g} / \mathrm{h}$, which means the substrate was being digested by enzyme very fast and the hydrolysis was going well.

The corresponding output membership functions for feeding time and feeding speed were defined to give proper responses to different input statuses. The combination of feeding time and speed was set to dose certain amount of pretreated biomass into hydrolysis system. The difficulty of determining how much pretreated biomass should be fed into system lies in the compositions of pretreated biomass and solids content of current system. Pretreated biomass contains $52.99 \%$ of cellulose which could be digested by enzyme to glucose and $33.31 \%$ lignin which cannot be digested and would accumulate in system. The detailed relationship between enzyme and compositions in pretreated biomass has been studied by Tai and Keshwani [19]. When a certain amount of cellulose was digested during a sample interval, which means the system would have the ability to digest same or similar amount of cellulose in the next sample interval, this amount of cellulose should be fed into system. However, with the extra lignin being fed with the biomass, solids content of current system would not be able to maintain constantly at $10 \%$, which in turn would decrease the ability of cellulose digestion [27]. While if an equivalent amount of biomass was chosen to be fed into the system to maintain solids content, the addition of cellulose available for digestion would be about $47.01 \%$ less than what the reaction needed.

Fuzzy logic control strategy successfully solved this dilemma through the settings in membership functions and rule-base. The range for feeding time was from 100 to 
Fig. 3 Block diagram (a) and front panel (b) of fuzzy logic control system in LabVIEW

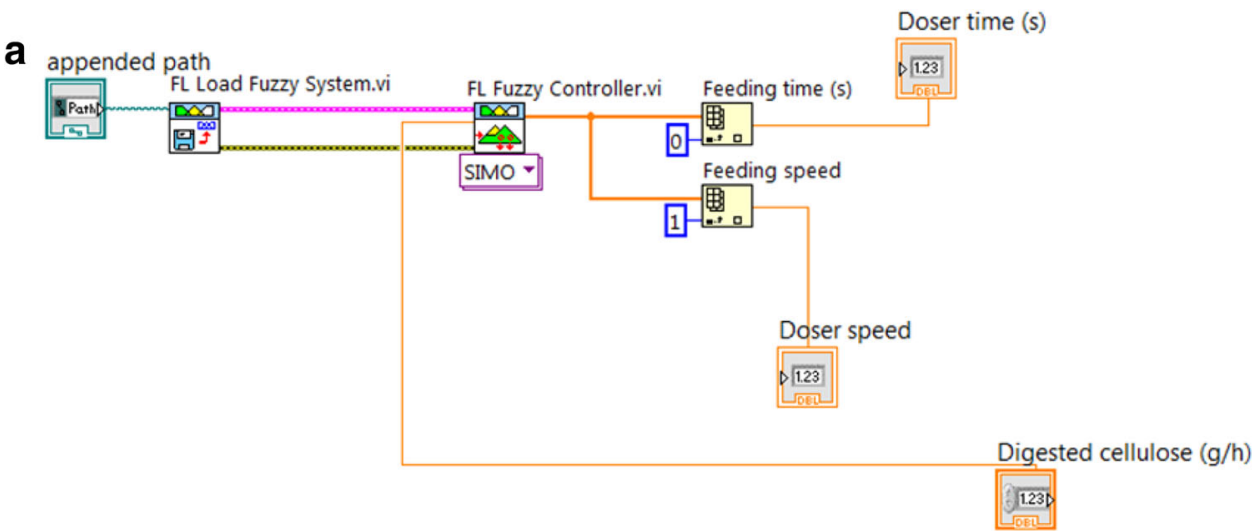

b

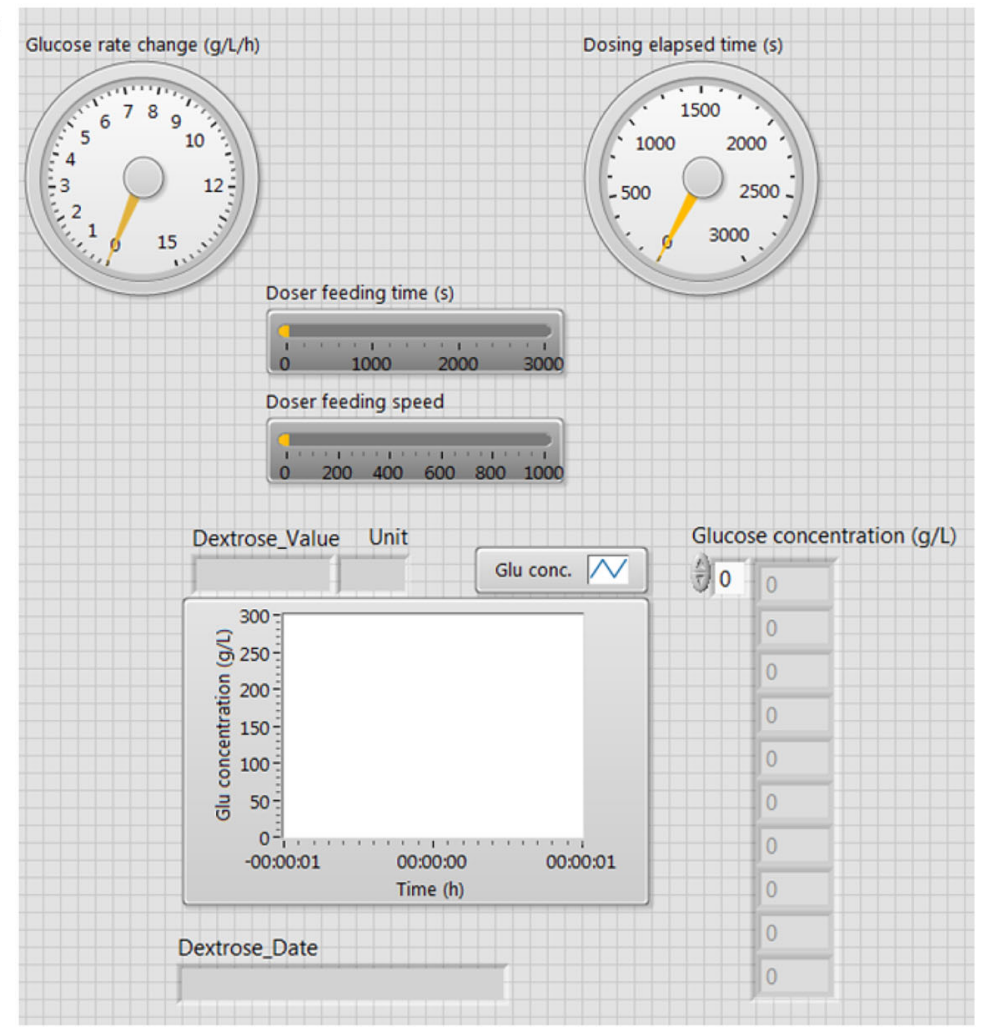

$2700 \mathrm{~s}$ while the feeding speed was from 100 to 999 (Fig. 2b, c). Feeding time and speed were divided into five and three categories, respectively. Every rule-base which combines the full membership in feeding time and speed would dose pretreated biomass which contains exactly same amount of cellulose as digested, while every rulebase which combines membership at cross points would dose pretreated biomass whose weight is equal to the digested cellulose in input.

The rule-base was created to build relationship between input status and output responses (Table 1). The rule-base was set in a way where multiple choices ("OR" relationship) for feeding time and speed would be available for system. For example, when input digested glucose was "High", the doser feeding time could be "Long" and speed could be "Fast", while feeding time could also be "Very Long" and speed could be "Medium", where both of the cases would dose the same amount of pretreated substrate, but in a different time period. When a fuzzified input falls in a certain category which leads to multiple choices of output in rule-base, all choices are being triggered and an average of them would be taken by the center of area defuzzification method with each degree of support. In our case, the degree of support for each rule is set to be 1 so no choice is favored. False case of negative digested glucose was created for the programming need. 


\section{System performance in fed-batch enzymatic hydrolysis}

The system was tested using the "Control design and simulation" tool in LabVIEW. The result of glucose accumulation in real application was shown in Fig. 4 and the accumulated cellulose conversion was shown in Fig. 5. The fuzzy logic controlled hydrolysis started with an initial solids loading of $10 \%$, the first sample was taken at $2 \mathrm{~h}$, where glucose concentration was $13.43 \mathrm{~g} / \mathrm{L}$, a little lower than in batch hydrolysis. The fuzzy logic program responded to this glucose concentration and $6.77 \mathrm{~g}$ of biomass was gradually fed into system in $1084 \mathrm{~s}$. With an initial system volume of $300 \mathrm{~mL}, 13.43 \mathrm{~g} / \mathrm{L}$ glucose means $3.63 \mathrm{~g}$ cellulose in substrate was digested, which further means the system at that moment has a digestion ability of 3.72-7.01 g dried pretreated biomass with moisture content of $2.38 \%$ and cellulose content of $52.99 \%$. It was clear that if $3.72 \mathrm{~g}$ biomass was added, the solids content of system will remain $10 \%$ while if $7.01 \mathrm{~g}$ biomass was added, the exact $3.63 \mathrm{~g}$ cellulose would be brought into system, but solids content would be up to $10.82 \%$. With the settings in membership functions and rule-base, $6.77 \mathrm{~g}$ appears to be a reasonable decision. The balance between bringing in equivalent amount of cellulose and maintaining solids content in system was considered in every decision after sampling.

With the feedings coming into system, as we can see in Fig. 4, the hydrolysis rate decreased compared to batch hydrolysis, indicating possible deficiencies in mass transfer and/or enzyme transition from partially degraded cellulose to new substrate. Even though there is new enzyme being fed into system, lignin is being accumulated in the system. Since lignin is a barrier to hydrolysis, its inhibition effect would accumulate over the hydrolysis time [24]. Yang et al. [28] showed fed-batch hydrolysis performance would be much better if multiple pretreatment methods have been used, where a major part of both xylan and lignin has been

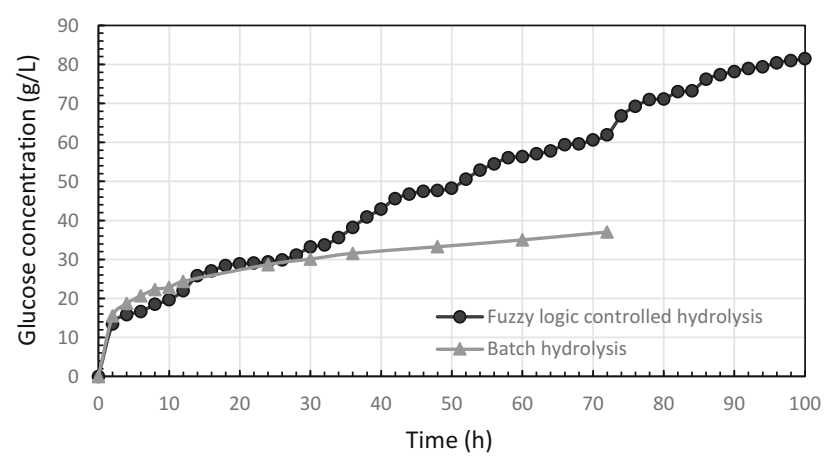

Fig. 4 Glucose accumulation during enzymatic hydrolysis of pretreated biomass

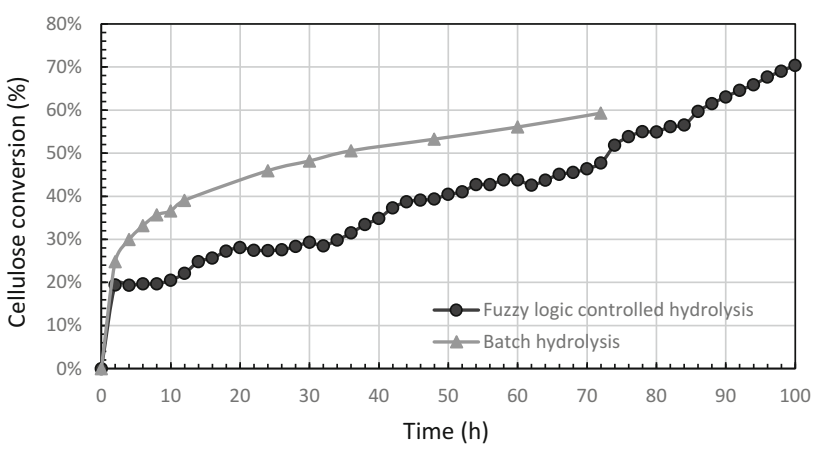

Fig. 5 Cellulose conversion during enzymatic hydrolysis of pretreated biomass

removed from substrate. There are also certain plateau phases during hydrolysis, such as 18-26 and 44-48 h, where cellulose digestion rate was very low and little glucose was released. These phases are probably caused by a feeding of relatively large amount of biomass which made solids content much higher and mass transfer not sufficient enough.

A total of 1554 FPU of enzyme and $150.48 \mathrm{~g}$ of pretreated biomass have been fed into system through 2-86 h. $19.96 \mathrm{FPU} / \mathrm{g}$ cellulose of enzyme has been maintained as average for feedings. As we can see in Fig. 5, $20 \mathrm{FPU} / \mathrm{g}$ cellulose of enzyme with $10 \%$ solids loading in batch hydrolysis would hydrolyze $59.29 \%$ of cellulose in substrate, while the accumulated cellulose conversion in fuzzy logic controlled fed-batch was $11.10 \%$ higher at $100 \mathrm{~h}$. Accumulated cellulose conversion was calculated based on NREL procedure [29] which involves determining the total grams of cellulose digested (based on glucose concentration in the hydrolysis supernatant) and dividing that by the grams of cellulose present in the total biomass. With pretreated biomass feeding into system, the accumulated cellulose conversion was not increasing sharply until the end of feeding at $86 \mathrm{~h}$. There were also certain times where the conversion was lower than the one before because of the relatively large amount of pretreated biomass fed into system at that time point.

Fuzzy logic controlled fed-batch enzymatic hydrolysis appeared to perform well in response to glucose concentration detected online. The system provides a feasible realtime monitor and control strategy for hydrolysis processes where information about reaction mechanisms and control indices are difficult to obtain and/or are ambiguous. Using a single online parameter (glucose concentration) and a rule-base derived from batch hydrolysis experiment, the system was able to make effective decisions on feeding substrate to maintain desirable performance in the fedbatch hydrolysis process. With the consideration of certain plateau phases, a more suitable rule-base or more detailed membership functions could be created to avoid feeding of 
excess pretreated biomass at some time points. Additionally, online determination of system viscosity would help to identify undigested substrate and lignin in the system, which would further improve the knowledge of input membership functions and the rule-base.

\section{Conclusions}

A fuzzy logic control system has been developed for online feeding controls in fed-batch enzymatic hydrolysis of dilute acid-pretreated corn stover. Compared to traditional controls, fuzzy logic provides the advantages of less input information and efficient real-time responses. Difficult model prediction for interference of added pretreated feedings was successfully avoided. The feeding operations were determined timely by fuzzy logic control system and effective responses were shown to properly deal with plateau phases during hydrolysis. Feeding of a proper amount of cellulose in biomass and maintaining solids content in system were well balanced. Fuzzy logic proved to be a robust and effective online feeding control tool for fedbatch enzymatic hydrolysis.

Acknowledgments This work was carried out with funding from a strategic research grant from the Institute of Agriculture and Natural Resources at the University of Nebraska. The authors would like to thank Novozymes North America Inc. for providing CTec2 and Mr. Aaron Engel for considerable help of LabVIEW programming.

\section{References}

1. Ragauskas AJ, Williams CK, Davison BH, Britovsek G, Cairney J, Eckert CA, Frederick WJ Jr, Hallett JP, Leak DJ, Liotta CL (2006) The path forward for biofuels and biomaterials. Science 311(5760):484-489

2. Tai C, Keshwani D (2014) Impact of pretreatment with dilute sulfuric acid under moderate temperature on hydrolysis of corn stover with two enzyme systems. Appl Biochem Biotechnol 172(5):2628-2639

3. Berndes G (2002) Bioenergy and water-the implications of large-scale bioenergy production for water use and supply. Global Environ Change 12(4):253-271. doi:10.1016/S09593780(02)00040-7

4. Klein-Marcuschamer D, Oleskowicz-Popiel P, Simmons BA, Blanch HW (2012) The challenge of enzyme cost in the production of lignocellulosic biofuels. Biotechnol Bioeng 109(4):1083-1087

5. Wingren A, Galbe M, Zacchi G (2003) Techno-economic evaluation of producing ethanol from softwood: comparison of SSF and SHF and identification of bottlenecks. Biotechnol Prog 19(4):1109-1117

6. Öhgren K, Bura R, Saddler J, Zacchi G (2007) Effect of hemicellulose and lignin removal on enzymatic hydrolysis of steam pretreated corn stover. Bioresour Technol 98(13):2503-2510. doi:10.1016/j.biortech.2006.09.003

7. Tai C, Keshwani DR, Voltan DS, Kuhar PS, Engel AJ (2015) Optimal control strategy for fed-batch enzymatic hydrolysis of lignocellulosic biomass based on epidemic modeling. Biotechnol Bioeng 112(7):1376-1382

8. Hodge D, Karim M, Schell D, McMillan J (2009) Model-based fed-batch for high-solids enzymatic cellulose hydrolysis. Appl Biochem Biotechnol 152(1):88-107. doi:10.1007/s12010-0088217-0

9. Rosgaard L, Andric P, Dam-Johansen K, Pedersen S, Meyer A (2007) Effects of substrate loading on enzymatic hydrolysis and viscosity of pretreated barley straw. Appl Biochem Biotechnol 143(1):27-40. doi:10.1007/s12010-007-0028-1

10. Khamseh AAG, Miccio M (2012) Comparison of batch, fedbatch and continuous well-mixed reactors for enzymatic hydrolysis of orange peel wastes. Process Biochem 47(11):1588-1594. doi:10.1016/j.procbio.2011.10.018

11. Chandra RP, Au-Yeung K, Chanis C, Roos AA, Mabee W, Chung PA, Ghatora S, Saddler JN (2011) The influence of pretreatment and enzyme loading on the effectiveness of batch and fed-batch hydrolysis of corn stover. Biotechnol Prog 27(1):77-85. doi: $10.1002 /$ btpr.508

12. Ros SD, Colusso G, Weschenfelder TA, de Marsillac Terra L, de Castilhos F, Corazza ML, Schwaab M (2013) A comparison among stochastic optimization algorithms for parameter estimation of biochemical kinetic models. Appl Soft Comput 13(5):2205-2214. doi:10.1016/j.asoc.2013.01.019

13. Regunath S, Kadirkamanathan V (2001) Design of a pH control system using fuzzy non-uniform grid scheduling and evolutionary programming. Appl Soft Comput 1(1):91-104. doi:10.1016/ S1568-4946(01)00011-4

14. Yager RR, Zadeh LA (2012) An introduction to fuzzy logic applications in intelligent systems. Springer, New York

15. Scherer P, Lehmann K, Schmidt O, Demirel B (2009) Application of a fuzzy logic control system for continuous anaerobic digestion of low buffered, acidic energy crops as mono-substrate. Biotechnol Bioeng 102(3):736-748. doi:10.1002/bit.22108

16. Traoré A, Grieu S, Puig S, Corominas L, Thiery F, Polit M, Colprim J (2005) Fuzzy control of dissolved oxygen in a sequencing batch reactor pilot plant. Chem Eng J 111(1):13-19. doi:10.1016/j.cej.2005.05.004

17. Wan J, Huang M, Ma Y, Guo W, Wang Y, Zhang H, Li W, Sun X (2011) Prediction of effluent quality of a paper mill wastewater treatment using an adaptive network-based fuzzy inference system. Appl Soft Comput 11(3):3238-3246. doi:10.1016/j.asoc. 2010.12.026

18. Tai C, Arellano MG, Keshwani DR (2014) Epidemic based modeling of enzymatic hydrolysis of lignocellulosic biomass. Biotechnol Prog 30(5):1021-1028

19. Tai C, Keshwani DR (2014) Enzyme adsorption and cellulose conversion during hydrolysis of dilute-acid-pretreated corn stover. Energy Fuels 28(3):1956-1961

20. Adney B, Baker J (1996) Measurement of cellulase activities. Laboratory analytical procedure, vol 6. National Renewable Energy Laboratory (NREL), Golden

21. Sluiter A, Hames B, Hyman D, Payne C, Ruiz R, Scarlata C, Sluiter J, Templeton D, Wolfe J (2008) Determination of total solids in biomass and total dissolved solids in liquid process samples. National Renewable Energy Laboratory (NREL), Golden

22. Sluiter A, Hames B, Ruiz R, Scarlata C, Sluiter J, Templeton D, Crocker D (2011) Determination of structural carbohydrates and lignin in biomass. National Renewable Energy Laboratory (NREL), Golden

23. Ross TJ (2009) Fuzzy logic with engineering applications. Wiley, New York

24. Kuhad RC, Mehta G, Gupta R, Sharma KK (2010) Fed batch enzymatic saccharification of newspaper cellulosics improves the sugar content in the hydrolysates and eventually the ethanol 
fermentation by Saccharomyces cerevisiae. Biomass Bioenergy 34(8):1189-1194. doi:10.1016/j.biombioe.2010.03.009

25. Wanderley MCdA, Martín C, Rocha GJdM, Gouveia ER (2013) Increase in ethanol production from sugarcane bagasse based on combined pretreatments and fed-batch enzymatic hydrolysis. Bioresour Technol 128:448-453. doi:10.1016/j.biortech.2012.10. 131

26. Morales-Rodríguez R, Capron M, Huusom JK, Sin G (2010) Controlled fed-batch operation for improving cellulose hydrolysis in $2 \mathrm{G}$ bioethanol production. Comput Aided Chem Eng 28:1497-1502
27. Kristensen J, Felby C, Jorgensen H (2009) Yield-determining factors in high-solids enzymatic hydrolysis of lignocellulose. Biotechnol Biofuels 2(1):11

28. Yang M, Li W, Liu B, Li Q, Xing J (2010) High-concentration sugars production from corn stover based on combined pretreatments and fed-batch process. Bioresour Technol 101(13):4884-4888

29. Selig M, Weiss N, Ji Y (2008) Enzymatic saccharification of lignocellulosic biomass. National Renewable Energy Laboratory (NREL), Golden 